\title{
Síndrome de resección anterior baja en pacientes con cáncer de recto medio e inferior ¿Qué más podemos hacer?
}

\author{
Carmen Paz Abarca ${ }^{1,3}$, Macarena Fernández A. 2,3
}

'Centro Integral de Reeducación de Piso Pélvico de Cirugía. Hospital Clínico San Borja Arriarán, Campus Centro, Facultad de Medicina Universidad de Chile.

${ }^{3}$ Comité Científico Sociedad Chilena de Uroginecología y Piso Pélvico (SODUP)

Recibido 2020-05-18 y aceptado 2020-06-29

Correspondencia a: Dra. Macarena Fernández A. contacto@

dramacarenafernandez.c

\section{Low anterior resection syndrome in patients with medium and low rectal} cancer. What else can we do?

During the last decades, advances in surgical techniques, radiotherapy and chemotherapy have significantly increased survival and reduced local recurrence in rectal cancer, avoiding a definitive colostomy. However, this transcendental medical progress has not been accompanied by an improvement in the functional results of patients after sphincter-preserving rectal resection, being the set of defecatory symptoms after rectal resection, known as low anterior resection syndrome (LARS), a practically inevitable sequel, which is usually associated with sexual or urinary dysfunction, chronic pain, high costs and an important alteration in the quality of life. In this article, we will present a complete and updated review of the clinical and alternative characteristics of LARS treatment, ending with a proposal for multidisciplinary and integral management that highlights the importance of prehabilitation, objective evaluation of symptoms, education and adequate information of the patient and his family, in addition to teamwork in units specialized in pelvic floor rehabilitation, a fundamental requirement when properly managing this pathology.

Key words: low anterior resection syndrome; rectal cancer; pelvic floor rehabilitation; LARS score; fecal incontinence.

\section{Resumen}

Durante las últimas décadas los avances en técnicas quirúrgicas, radioterapia y quimioterapia han logrado de forma significativa aumentar la sobrevida y disminuir la recidiva local en el cáncer de recto evitando una colostomía definitiva; sin embargo, este trascendental progreso médico no ha ido acompañado de una mejoría en los resultados funcionales de los pacientes sometidos a una cirugía conservadora de esfínter, siendo el conjunto de síntomas defecatorios posterior a la resección rectal, conocido como síndrome de resección anterior baja (LARS), una secuela prácticamente inevitable, que generalmente se asocia a disfunción sexual, urinaria, dolor crónico, altos costos en rehabilitación y control sintomático, además de una alteración importante en la calidad de vida. En este artículo presentamos una revisión completa y actualizada de las características clínicas y alternativas de tratamiento del LARS, finalizando con una propuesta de manejo integral multidisciplinario que destaca la importancia de la prehabilitación, evaluación objetiva de los síntomas, educación e información adecuada del paciente y su familia, además del trabajo en equipo en unidades especializadas de rehabilitación de piso pélvico, requisito fundamental a la hora de manejar adecuadamente esta patología.

Palabras clave: síndrome resección anterior baja; cáncer de recto; rehabilitación de piso pélvico; LARS score; incontinencia fecal.

\section{Introducción}

Uno de los avances más relevantes en cirugía colorrectal es el uso creciente de procedimientos con preservación de esfínter, siendo actualmente la resección anterior baja (RAB) más la escisión total del mesorrecto (ETM) el tratamiento estándar para la mayoría de los pacientes portadores de un cáncer de recto medio e inferior, además de la resección anterior ultrabaja (RAUB) y resección anterior ultrabaja interesfintérica (RAUBIE) en casos seleccionados ${ }^{1}$. 
Superada la mutilación de la colostomía definitiva aparece el problema de la disfunción intestinal y su impacto en la calidad de vida, lo que se conoce como síndrome de resección anterior baja (LARS), problema frecuente, subvalorado, diagnosticado tardíamente, con herramientas recientemente validadas y tratamiento basado en evidencia científica escasa y heterogénea.

El objetivo de esta revisión es sintetizar la evidencia bibliográfica actualizada acerca del LARS y plantear un algoritmo de intervención terapéutica interdisciplinaria en las distintas etapas de la enfermedad en pacientes portadores de un cáncer de recto medio e inferior, sometidos a una RAB y sus variantes.

\section{Materiales y Método}

Se realizó una revisión bibliográfica en PubMed utilizando los términos mesh: "Low anterior resection syndrome", "Rectal cancer", "Pelvic floor rehabilitation", "Biofeedback", "LARS score", "Fecal incontinence", "Treatment”, "Rehabilitation". Se consideró la combinación de términos presentes en palabras clave y en los títulos de las publicaciones. Se incluyeron revisiones sistemáticas con y sin metaanálisis, estudios de cohortes retrospectivos y prospectivos, estudios de casos y controles y ensayos clínicos randomizados, publicados entre los años 2008 y 2019 en idioma inglés y español. No hubo restricción en cuanto a tamaño de muestra.

\section{Definición}

No existe una definición de consenso para el LARS. Actualmente, se considera que corresponde a un conjunto amplio de síntomas intestinales que se manifiestan posterior a la cirugía rectal conservadora de esfínter, técnica quirúrgica que hoy se realiza en más del $80 \%$ de los pacientes portadores de un cáncer de recto ${ }^{2}$.

\section{Epidemiología}

La evidencia disponible indica que el LARS se presenta en más de $90 \%$ de los casos, en el $41 \%$ de forma severa ${ }^{3}$ y cerca del $50 \%$ mantiene la disfunción intestinal 14 años posterior a la intervención quirúrgica ${ }^{4}$.

Posterior a la resección rectal por cáncer el $38,8 \%$ de los casos vive con dolor crónico en la pelvis o en extremidades inferiores ${ }^{5}$ y el $10 \%-35 \%$ presenta síntomas urológicos y/o sexuales asocia$\operatorname{dos}^{6}$ (incontinencia urinaria, disfunción eréctil, eyaculación retrógrada y dispareunia). El 30\%-40\% de los pacientes que relatan ser sexualmente activos en el preoperatorio se vuelve inactivo posterior a la cirugía 7 .

\section{Fisiopatología}

La fisiopatología exacta del LARS no está bien establecida. El origen multifactorial de este síndrome se puede dividir en tres pilares principales: disfunción del neorecto, dismotilidad colónica y alteración esfinteriana ${ }^{8-10}$ (Figura 1).

\section{Características clínicas y evaluación}

Una revisión sistemática sobre LARS de 128 estudios publicados entre 1986 y 2016 reveló la existencia de más de 30 síntomas, los reportados con mayor frecuencia fueron incontinencia fecal (97\%), aumento de la frecuencia de las deposiciones $(80 \%)$, urgencia defecatoria (67\%), disfunción evacuatoria (47\%) y alteración en la discriminación entre gases $y$ heces $(34 \%)^{11}$.

Como clínicos tendemos a clasificar al LARS en dos categorías, los pacientes con incontinencia fecal, frecuencia defecatoria aumentada y urgencia, y, por otra parte, los pacientes constipados y con sensación de vaciamiento incompleto. Esta clasificación no es válida ya que un porcentaje importante presenta ambos grupos de síntomas.

La mayoría de las publicaciones referentes al LARS no utiliza score, y en los trabajos que usan un método objetivo de valoración, el utilizado con mayor frecuencia es la escala de Wexner, confirmando la tendencia a sobreestimar la incontinencia anal, dejando sin consignar síntomas altamente incapacitantes como el clustering (evacuación incompleta que requiere volver al baño en un plazo inferior a 1 hora desde la vez anterior) y la urgencia defecatoria ${ }^{11}$

En este contexto, Emmertsen $\mathrm{K}$ et al. ${ }^{12}$, desarrollan el LARS score, considerado hoy el mejor instrumento de evaluación de este síndrome, por la directa correlación entre el puntaje asignado y su impacto en la calidad de vida. Es un cuestionario rápido, de fácil comprensión, que consta de cinco ítems, otorgando mayor relevancia a los síntomas de clustering y urgencia defecatoria, clasificando a los pacientes en LARS mayor, LARS menor y sin 
Figura 1. Fisiopatología multifactorial del LARS. EAl: Esfínter anal interno. EAE: Esfínter anal externo.

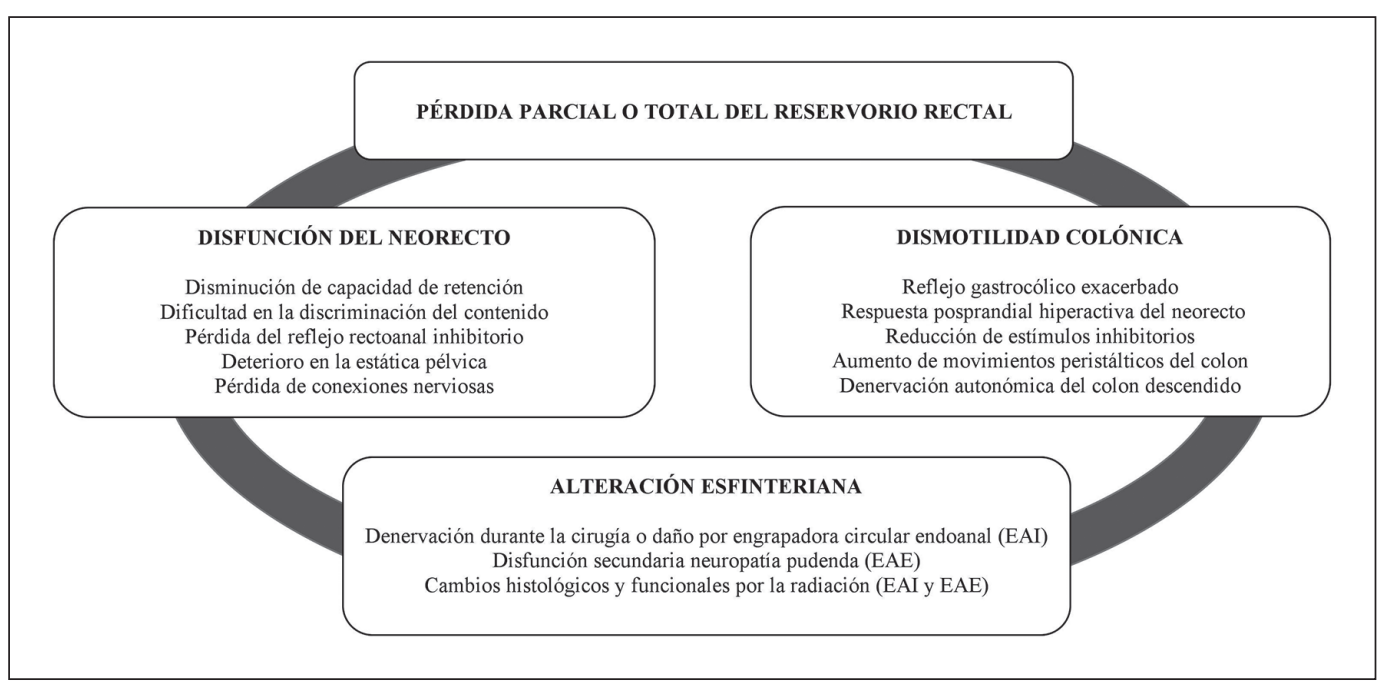

Figura 2. Adaptación cultural en español del LARS score.

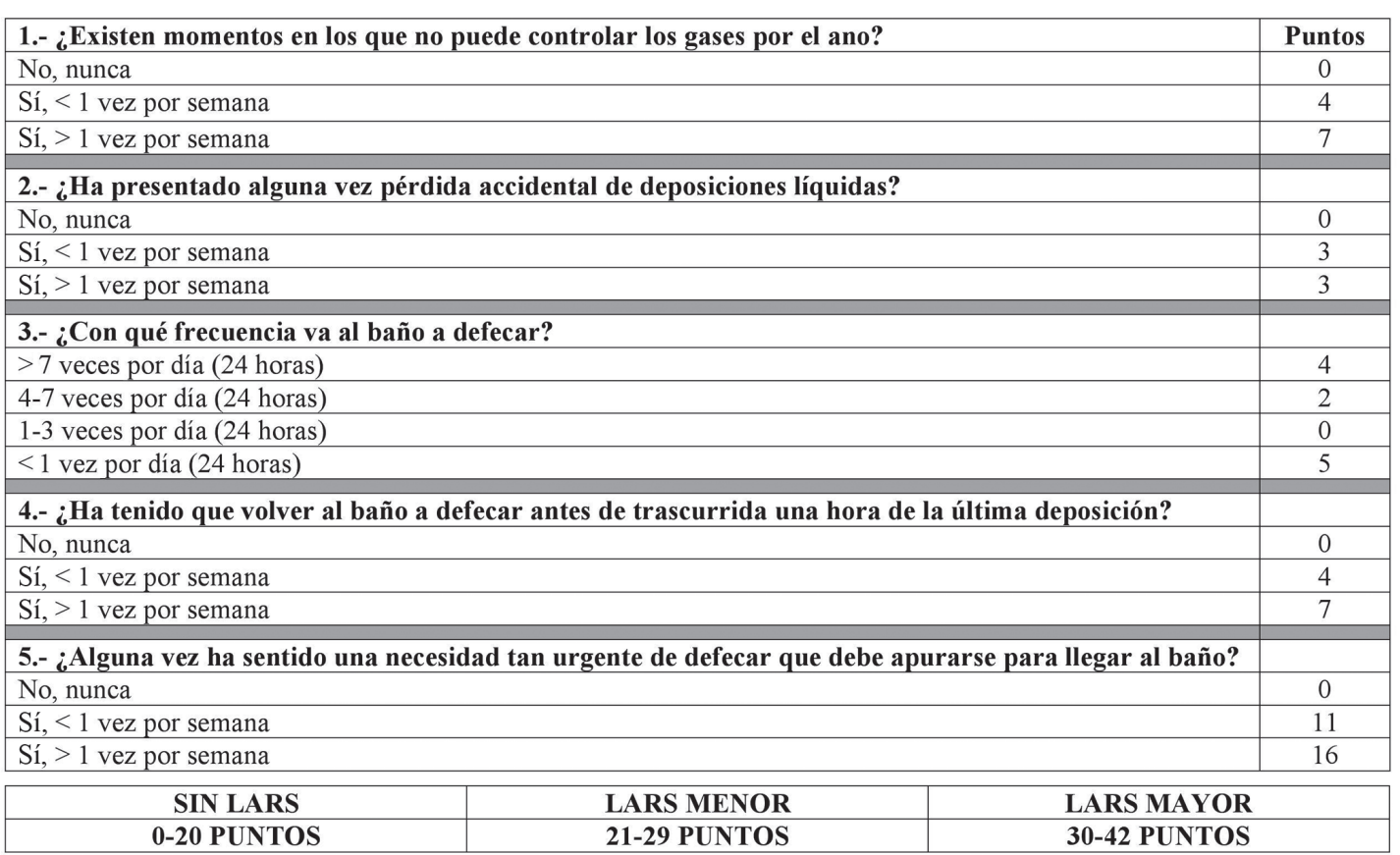

LARS. Actualmente contamos con una adaptación transcultural idiomática validada al español-chileno que puede ser aplicada a nuestra realidad local ${ }^{13}$ (Figura 2).

\section{Factores de riesgo}

Los pacientes con mayor probabilidad de padecer LARS son los menores de 65 años, de sexo feme- nino, con historia de radioterapia neoadyuvante, antecedente de tumor rectal o anastomosis colorrectal a menos de $5 \mathrm{~cm}$ del margen anal, necesidad de ileostomía derivativa de protección e historia de sepsis pélvica secundaria a dehiscencia anastomótica $^{3}$. Con el objetivo de predecir la gravedad del LARS antes de la resección rectal, Battersby et al. ${ }^{14}$, diseñaron el POLARS score basado en los factores de riesgo ya descritos (Figura 3). 


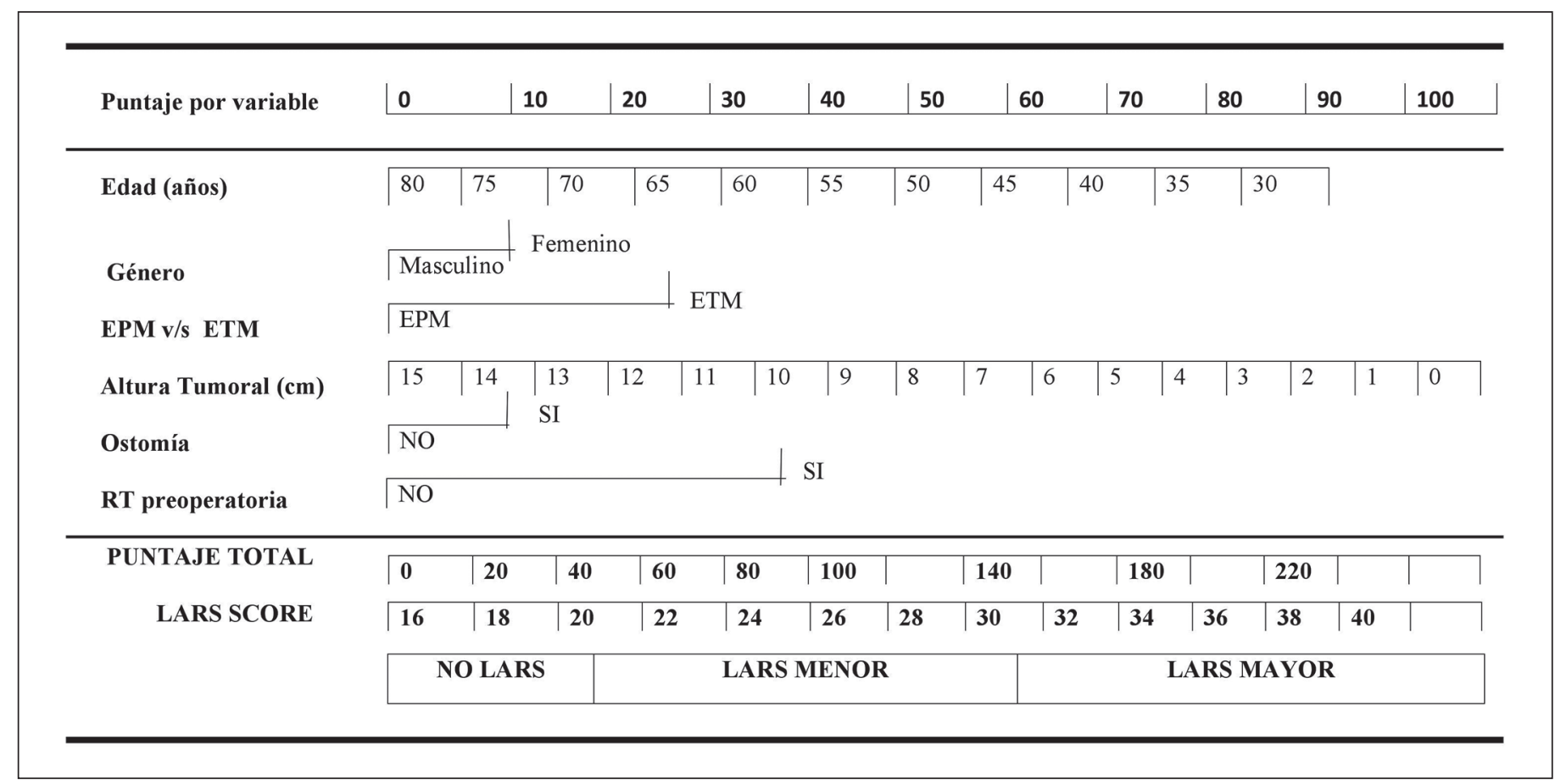

Figura 3. POLARS score. EPM: escisión parcial del mesorrecto. ETM: escisión total del mesorrecto. RT: radioterapia.

\section{Tratamiento}

No existe un tratamiento específico para el LARS. Generalmente tratamos los síntomas predominantes de forma empírica en base a las medidas usadas en otras disfunciones defecatorias, consistentes en cambios en la alimentación, suplementos de fibra, supositorios de glicerina, laxantes y enemas.

\section{Medidas farmacológicas}

\section{Loperamida}

Opioide ampliamente usado en incontinencia fecal. Reduce la actividad del plexo mientérico, suprime la respuesta gastrocólica, prolonga el tránsito intestinal, reduce el volumen fecal y aumenta el tono del esfínter anal. Sin embargo, hasta la fecha, no hay ningún ensayo clínico disponible que evalúe su uso específicamente en pacientes con LARS.

\section{Romasentron}

Potente y selectivo antagonista del receptor de serotonina 5-HT3, utilizado en el síndrome de intestino irritable con diarrea como síntoma predominante. Un estudio reciente en LARS muestra una reducción significativa de la frecuencia y urgencia defecatoria al administrar Romasentron 5 ug al día durante los primeros 6 meses posterior a la cirugía ${ }^{15}$; sin embargo, este medicamento aún no está disponible en nuestro medio.

\section{Irrigación retrógrada transrectal (IRT)}

Consiste en administrar 500 a $1.500 \mathrm{cc}$ de suero fisiológico 2-3 veces por semana a través de una sonda rectal diseñada para vaciar el recto y el colon distal. El paciente reduce el número de defecaciones diarias, episodios de incontinencia y evacuaciones nocturnas, mejorando su calidad de vida ${ }^{16}$. Recientemente, Martellucci et al., en un estudio de 27 pacientes con LARS tratados con IRT, logran una reducción del LARS score de 35,1 a 12,2 puntos después de 6 meses. Teóricamente la IRT tendría un efecto en la dismotilidad colónica, entregando un ritmo al tránsito colónico; además, a largo plazo podría aumentar el volumen rectal antes de desencadenar la urgencia ${ }^{17}$.

\section{Neuromodulación}

La neuromodulación central o sacra (NMS) a través de la estimulación eléctrica de las raíces S3 


\section{ARTíCULOS DE REVISIÓN}

y S4 genera la modulación de respuestas motoras, sensitivas y cognitivas asociadas a la defecación a través de modificaciones en la contractilidad, sensibilidad y distensibilidad rectal, disminución de la actividad anterógrada del colon y mejoría de la actividad muscular esfinteriana ${ }^{18}$. La eficacia de la NMS usada para incontinencia anal por LARS es comparable a los resultados de NMS usada para cualquier causa de incontinencia fecal ${ }^{19}$, además, sería una terapia efectiva para síntomas como fragmentación, urgencia y clustering ${ }^{20}$; sin embargo, la evidencia publicada aún es escasa (Tabla 1).

En cuanto a la neuromodulación periférica del nervio tibial posterior (PTNS), dos estudios prospectivos observacionales demostraron mejoría en la incontinencia fecal y disminución significativa del LARS score después de 12 sesiones de tratamiento ${ }^{21,22}$.

\section{Rehabilitación multimodal del piso pélvico}

La rehabilitación de piso pélvico (RPP), realizada por kinesiólogos con formación especializada en el área, es económica, no invasiva, con escasos efectos adversos y no excluye otros tratamientos ${ }^{21}$. Su objetivo en pacientes con LARS es reducir o eliminar las disfunciones defecatorias, urinarias y sexuales, además del manejo del dolor y mejorar la calidad de vida $^{23,24}$. Las diferentes técnicas kinésicas utilizadas en RPP se describen en la Tabla 2.

Una revisión sistemática demostró la utilidad de la RPP para mejorar el resultado funcional después de una RAB. De 5 estudios incluidos (321 pacientes) el $90 \%$ utilizó entrenamiento muscular de piso pélvico (PP) y en 4, se agregó entrenamiento con BFB muscular o rectal. La mayoría de los estudios mostraron una mejoría en la continencia, frecuencia de deposiciones y la calidad de vida en general $^{25-30}$.

Aunque en la mayoría de los estudios no se especifica el tipo de biofeedback (BFB) utilizado, se concluye que es un procedimiento seguro, no invasivo, barato y exitoso en el tratamiento de pacientes con LARS $^{27-29}$. Liang et al..$^{30}$, demuestran que el uso de BFB muscular y rectal tienen un efecto positivo sobre la continencia, frecuencia defecatoria, todos los parámetros de la manometría anorrectal, la sensibilidad rectal y la calidad de vida en pacientes con LARS.

Por otra parte, Kye et al. ${ }^{27}$ plantean que un factor relacionado con la urgencia defecatoria es la hipersensibilidad rectal, y que el entrenamiento de PP combinado con BFB logra prevenir o minimizar esta alteración mejorando la presión anal de reposo, por lo tanto, sería esencial mantener la función del esfínter anal interno en condiciones en las que la compliance rectal disminuye después de la resección rectal. Los ejercicios de PP no supervisados (conocidos como Kegel) no garantizan la ejecución correcta a diferencia del BFB verificado y optimizado por especialista.

\section{Prehabilitación}

La prehabilitación del cáncer es el proceso de continuidad de la atención entre el momento del diagnóstico y el inicio del tratamiento, incluye evaluaciones físicas y psicológicas que establecen un nivel funcional basal, identifican deficiencias y

Tabla 1. LARS tratado con NMS: Comparación de series publicadas

\begin{tabular}{|lcccccccc|}
\hline Estudio & & $\mathbf{n}$ & $\begin{array}{c}\text { Wexner } \\
\text { Pre NMS }\end{array}$ & $\begin{array}{c}\text { Wexner } \\
\text { Post NMS }\end{array}$ & $\mathbf{p}$ & $\begin{array}{c}\text { LARS score } \\
\text { Pre NMS }\end{array}$ & $\begin{array}{c}\text { LARS score } \\
\text { Post NMS }\end{array}$ & $\begin{array}{c}\text { peguimiento } \\
\text { (meses) }\end{array}$ \\
Matzel & $(2002)$ & 1 & 17 & 2 & - & - & - & - \\
Jarret & $(2005)$ & 3 & - & - & - & - & - & - \\
Ratto & $(2005)$ & 4 & 16,3 & 4,5 & $<0,05$ & - & - & - \\
Holzer & $(2008)$ & 7 & 13 & 5 & - & - & - & - \\
De Miguel & $(2011)$ & 15 & 19,2 & 6,2 & $<0,01$ & - & - & - \\
Moya & $(2012)$ & 4 & 15,5 & 5,5 & $<0,005$ & - & - & - \\
Schwandner & $(2013)$ & 9 & 18,2 & 6,0 & $<0,01$ & - & - & - \\
D'Hondt & $(2017)$ & 11 & 17,7 & 4,6 & $<0,05$ & 36,9 & 12 & - \\
\hline Saleh & $(2017)$ & 12 & - & - & - & 34,0 & 21,6 & - \\
\hline
\end{tabular}

LARS: síndrome de resección anterior baja. NMS: neuromodulación sacra. 
Tabla 2. Herramientas de la rehabilitación de piso pélvico

\section{Técnicas de RPP}

1. Ejercicio terapéutico

2. Biofeedback muscular

4. Electroestimulación

\section{Definición - Objetivo}

Actividad física programada, estructurada y repetitiva con el objetivo de mejorar o mantener el rendimiento físico Herramienta kinesiológica que informa al paciente con señales visuales, auditivas y/o sensitivas sobre eventos fisiológicos para objetivar y optimizar las deficiencias mediante retroalimentación visual, electromiográfica o manométrica

\section{Herramienta kinesiológica que mediante técnicas de balón permiten mejorar la sensibilidad, acomodación rectal,} capacidad del neorecto, reflejos, sincronismo anorrectal y la evacuación

Herramienta instrumental que a través de la emisión de corrientes eléctricas potencia los efectos de la terapia kinésica
A) Estimulación funcional: mejora la propiocepción perineal, la contracción y tonificación muscular
B) Electroanalgesia: manejo sintomático del dolor
C) Neuromodulación del nervio tibial posterior (PTNS) ${ }^{25}$ : su mecanismo de acción sería similar al de la NMS

5. Terapia manual ortopédica

Estrategias manuales orientadas al manejo de alteraciones neuromusculoesqueléticas que potencian los efectos de la terapia kinésica (por ejemplo: masoterapia y liberación miofacial)

6. Entrenamiento muscular de PP

Programa terapéutico destinado a lograr correcta localización y disociación del piso pélvico, además de optimizar las cualidades musculares del MEA y EAE, utilizando las herramientas kinésicas antes mencionadas

7. Entrenamiento funcional Estrategia de intervención cuyo objetivo es estimular y automatizar la contracción del piso pélvico ante situade PP ciones de esfuerzo o aumento de la presión intraabdominal y reestablecer el sincronismo del sistema toracoabdómino-pélvico

8. Tratamiento conductual Herramienta kinesiológica que a través de la educación y orientación tiene el objetivo de cambiar patrones de comportamiento que están alterados como, por ejemplo, hábitos alimenticios, de evacuación y posturales; como también orientar en el uso de facilitadores del desempeño funcional

RPP: rehabilitación de piso pélvico. MEA: músculo elevador del ano. EAE: esfínter anal externo. PP: piso pélvico. NMS: neuromodulación sacra. brindan intervenciones dirigidas a mejorar la salud y reducir la incidencia y severidad de las alteraciones actuales y futuras ${ }^{31,32}$.

Publicaciones recientes han descrito numerosos beneficios para el bienestar posoperatorio con programas de prehabilitación en cáncer colorrectal, demostrando que pueden mejorar o mantener la capacidad funcional preoperatoria, lo que impacta positivamente la recuperación postoperatoria ${ }^{33-35}$. Otros estudios han demostrado que realizar una intervención de prehabilitación antes de la terapia neoadyuvante es una intervención factible, segura, no compromete la vía de tratamiento planificada y reduce el deterioro de la condición física ${ }^{36-40}$. Estudios informan sobre prehabilitación de PP en cáncer de recto antes del cierre de ileostomía con resultados promisorios (Tabla 3 ).

\section{Pre-habilitación en LARS y calidad de vida}

El estudio cualitativo de Van der Heijden et al. ${ }^{41}$, tuvo por objetivo dilucidar cómo el LARS afecta la calidad de vida desde la perspectiva del paciente con cáncer rectal para mejorar la estrategia de atención actual. Como resultado se identificaron tres temas principales que se describen en la Tabla 4. Se concluye que, la asesoría y conocimiento preoperatorio más el tratamiento posoperatorio precoz del LARS son esenciales, que la satisfacción con la información y la participación activa del paciente conducen a una mejor calidad de vida y mejores resultados psicosociales, y que los programas de prehabilitación pueden ser la estrategia más prometedora en la literatura actual.

\section{Intervención multidisciplinaria avanzada en LARS para pacientes con cáncer de recto medio e inferior}

En base a la evidencia expuesta planteamos un flujograma de manejo integral avanzado para pacientes con LARS secundario a cáncer de recto medio e inferior (Figura 4). 


\section{ARTÍCULOS DE REVISIÓN}

Tabla 3. Prehabilitación de piso pélvico en pacientes con cáncer de recto sometidos a cirugía conservadora de esfínter

\begin{tabular}{|c|c|c|c|c|}
\hline Estudio & n & Outcome & Intervención & Resultados \\
\hline $\begin{array}{l}\text { Kim } \\
(2015) \\
\text { Prospectivo } \\
\text { randomizado }\end{array}$ & 16 & $\begin{array}{l}\text { Manometría anal, SW, FI-QoL y } \\
\text { Bristol para patrón defecatorio }\end{array}$ & $\begin{array}{l}\text { Terapia conservadora (ejercicios } \\
\text { no supervisados y terapia } \\
\text { conductual) versus terapia de BFB } \\
\text { más terapia conductual previo a } \\
\text { cierre de ileostomía }\end{array}$ & $\begin{array}{l}\text { Ambos grupos mejoran SW y CV. } \\
\text { El grupo con BFB mejora } \\
\text { significativamente la presión } \\
\text { máxima de cierre tras } 12 \text { meses de } \\
\text { la cirugía. Concluyen que ambas } \\
\text { terapias son beneficiosas para } \\
\text { tratar la disfunción anorrectal en } \\
\text { pacientes con LARS }\end{array}$ \\
\hline $\begin{array}{l}\text { Kye } \\
(2016) \\
\text { Prospectivo } \\
\text { randomizado }\end{array}$ & 56 & $\begin{array}{l}\text { Manometría rectal, CCIS, } \\
\text { SW, defecaciones/día, uso de } \\
\text { loperamida y Cuestionario de } \\
\text { severidad de IF }\end{array}$ & $\begin{array}{l}\text { BFB versus ejercicios de Kegel } \\
\text { (sin supervisión) durante el } \\
\text { período del estoma temporal }\end{array}$ & $\begin{array}{l}\text { Logra evidenciar efecto } \\
\text { significativo sobre presión anal } \\
\text { de reposo } 6 \text { meses posterior a la } \\
\text { cirugía de conservación de esfínter } \\
\text { en el grupo de BFB versus el } \\
\text { grupo control }\end{array}$ \\
\hline
\end{tabular}

SW: score de Wexner. FI-QOL: escala de calidad de vida en incontinencia fecal. CCIS: Cleveland Clinic Incontinence Score. BFB: biofeedback. RPP: rehabilitación de piso pélvico. PP: piso pélvico. CV: calidad de vida.

Tabla 4. LARS y calidad de vida

Feedback de los participantes
Percepción de la enfermedad
1. Necesidad de atención específica a la presencia de síntomas:
Romper el taboo del LARS
2. Necesidad de mayor información acerca de curso normal
posoperatorio
Apoyo de cuidados preoperatorio
1. Subestimación del grado de invalidez que puede causar el LARS
2. El contacto personal sigue siendo la opción preferida
3. El apoyo social es importante y no siempre se puede asumir que
está presente
4. Poca motivación intrínseca para los programas de entrenamiento
antes de la cirugía, alta motivación intrínseca antes de la reversión
del estoma

\section{Apoyo de cuidados posoperatorio}

1. Los sentimientos de inseguridad y desesperación se identifican como el factor más importante que perjudica la calidad de vida en el período inmediatamente posterior al alta. Los pacientes sienten que hay una brecha en la atención de apoyo después del alta

\section{Cambios planificados o sugeridos}

\section{Percepción de la enfermedad}

1. Aumentar el conocimiento sobre LARS entre los especialistas con la implementación de herramientas de detección sistemática

2. Más información sobre el curso posoperatorio normal

\section{Apoyo de cuidados preoperatorio}

1+2. Mejor preparación sobre la posible carga de los síntomas de LARS. Los pacientes prefieren una película educativa a una aplicación

3. Actitud activa para discutir el impacto de los síntomas y para reforzar el apoyo social

4. Se desea contar con evidencia de vanguardia para programas de entrenamiento en pacientes con resección anterior baja con medidas de resultados funcionales. Se sugiere la RPP

\section{Apoyo de cuidados posoperatorio}

1. Introducción de una consulta telefónica con una enfermera de atención colorrectal 3 días después del alta

2. Participación adecuada del médico general para garantizar la derivación adecuada de los pacientes con LARS 

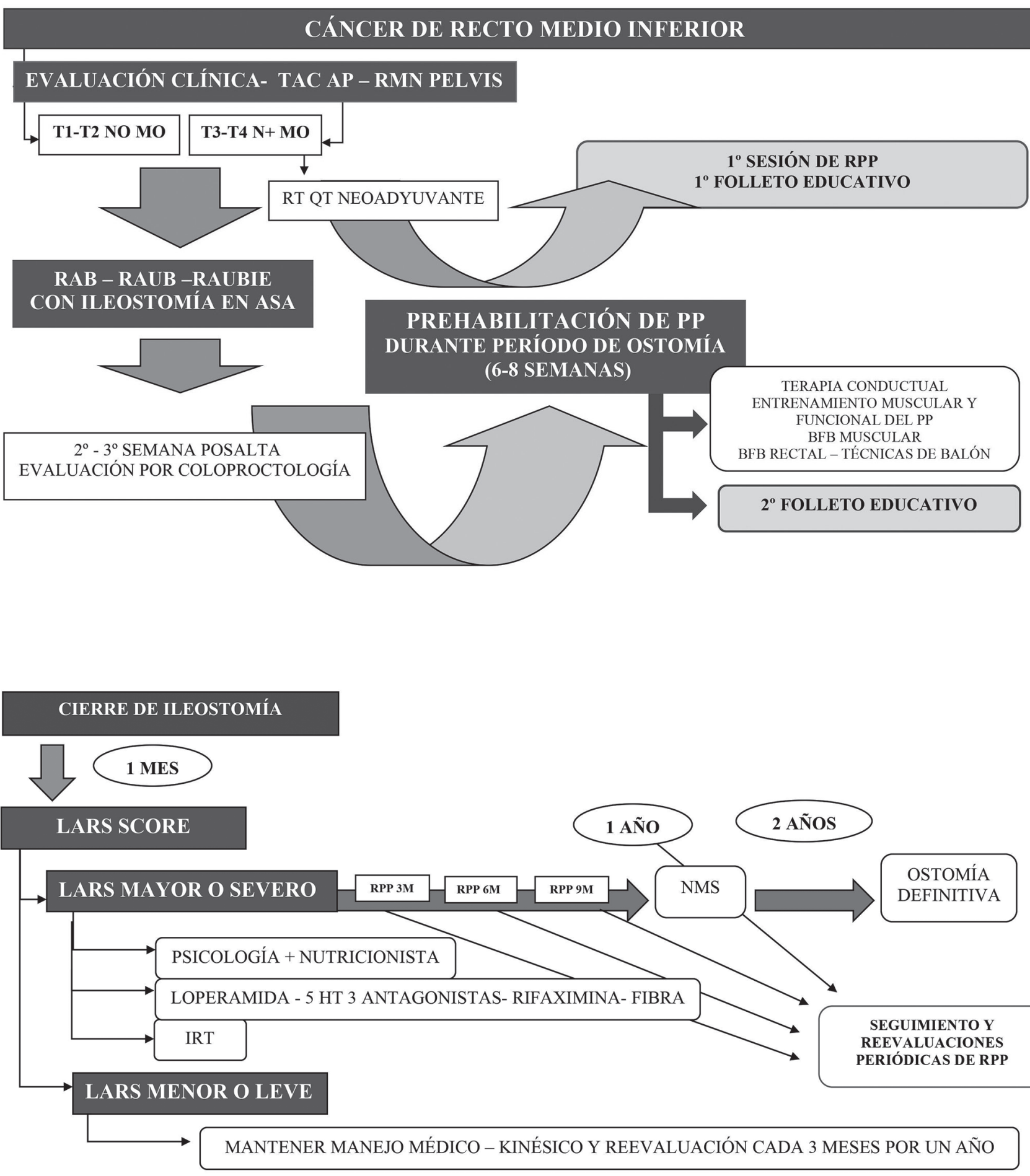

Figura 4. A y B: Intervención multidisciplinaria avanzada en LARS en pacientes con cáncer de recto medio e inferior. TAC AP: tomografía axial computada de abdomen y pelvis. RMN: resonancia nuclear magnética. RT: radioterapia. QT: quimioterapia. RAB: resección anterior baja. RAUB: resección anterior ultrabaja. RAUBIE: resección anterior ultrabaja interesfintérica. RPP: rehabilitación de piso pélvico. PP: piso pélvico. IRT: irrigación retrógrada transrectal. NMS: neuromodulación sacra. 
En este protocolo serán incluidos todos los pacientes con diagnóstico de cáncer de recto medio o inferior con o sin indicación de quimiorradioterapia neoadyuvante sometidos a cirugía preservadora de esfínter que requieran una ileostomía derivativa temporal: RAB, RAUB y RAUBIE. Serán excluidos los pacientes que presenten metástasis a distancia, disfunción cognitiva y complicaciones posoperatorias severas que requieran hospitalización mayor a 30 días.

Previo a la intervención quirúrgica, los pacientes que cumplan con los criterios de inclusión serán derivados por coloproctología a la primera sesión de RPP, donde se evaluará el estado basal de la región lumbo abdómino pélvica, perineal y anorectal incluyendo las cualidades musculares del PP, además de la presencia y severidad de disfunciones urinarias, defecatorias, sexuales, dolor y calidad de vida. Se entregará el $1^{\circ}$ folleto educativo con la siguiente información:

- Diagnóstico de cáncer de recto.

- Tratamiento quirúrgico.

- Concepto de PP.

- Primera pauta de ejercicios de PP.

- Primera pauta de ejercicios globales y respiratorios.

- Síntomas de alarma: dolor, fiebre, hemorragia, obstrucción.

Posterior a la resección rectal, 2-3 semanas después del alta hospitalaria, el paciente será reevaluado por coloproctología; si se encuentra en buenas condiciones generales y sin evidencia de complicación posoperatoria se derivará al Programa de Prehabilitación durante el período de 8-12 semanas que se mantenga ostomizado. Este programa consistirá en 10 sesiones de RPP distribuidas en una sesión semanal durante las primeras 6 semanas y 2 sesiones semanales las últimas 2 semanas previas a la reconstitución del tránsito intestinal. Las herramientas kinésicas utilizadas serán la terapia conductual, el entrenamiento muscular y funcional de PP y el BFB muscular. Luego del primer mes posoperatorio se adicionará el BFB rectal con técnicas de balón y la electroestimulación. En esta etapa del algoritmo se entregará el $2^{\circ}$ folleto educativo reforzando la importancia de la participación individual activa del paciente en su tratamiento contemplando:

- Objetivos del programa de prehabilitación.

- Refuerzo de información sobre PP.

- Pauta de progresión de entrenamiento muscular y funcional de PP.

- Segunda pauta de ejercicios globales y respiratorios.
- Posibles secuelas posoperatorias: LARS, dolor, disfunción sexual y urinaria.

- Medidas básicas para un buen manejo de la ostomía.

- Medidas de refuerzo y apoyo social.

Después de un mes de realizado el cierre de ileostomía, se calculará el LARS score basal. Mientras que a los pacientes sin LARS sólo se realizará seguimiento, los con LARS leve mantendrán el manejo médico-kinésico habitual que consiste en:

- 10 sesiones de RPP utilizando mismas herramientas kinésicas de la prehabilitación adicionando en esta etapa la PTNS.

- Seguimiento y reevaluaciones periódicas cada 3 meses por un año (incluyendo LARS score).

Por otro lado, los pacientes con LARS mayor (con diagnóstico inicial o en cualquier etapa del seguimiento) continuarán el manejo multimodal que consistente en:

- RPP descrita anteriormente durante mínimo 3 meses con reevaluaciones a los 3, 6, 9 y 12 meses (incluyendo LARS score).

- Evaluación y manejo por nutrición y psicología.

- Medidas farmacológicas, suplementos de fibra o IRT, según síntomas predominantes.

Si al completar un año de tratamiento médico el paciente es clasificado como LARS severo se evaluará la posibilidad de NMS. Si a los dos años el paciente mantiene un LARS severo, se considerará la ostomía definitiva como alternativa. Los pacientes que durante este período sean clasificados como LARS menor o sin LARS mantendrán las medidas no invasivas hasta ese momento efectivas más controles esporádicos.

\section{Conclusión}

Al explorar la bibliografía sobre las alternativas terapéuticas en pacientes con LARS, posterior al tratamiento quirúrgico de un cáncer de recto, podemos concluir que la evidencia es escasa y de mala calidad. No existe un protocolo de prevención ni manejo activo establecido para este tipo de pacientes.

Se propone un algoritmo piloto de intervención interdisciplinaria temprana que puede ser un aporte al implementarse y evaluar sus resultados, ya que no presenta efectos adversos ni mayores costos asociados, y podría mejorar considerablemente la calidad de vida de los pacientes portadores de un cáncer rectal que se someten a una proctectomía. 


\section{Responsabilidades éticas}

Protección de personas y animales. Los autores declaran que para esta investigación no se han realizado experimentos en seres humanos ni en animales.
Confidencialidad de los datos. Los autores declaran que en este artículo no aparecen datos de pacientes.

Conflictos de interés: no hay.

\section{Bibliografía}

1. Carrillo K, Abedrapo M, Azolas R. Técnicas quirúrgicas para la preservación de esfínter en cáncer de recto bajo: revisión histórica y estado actual. Rev Cir. 2018;70:178-84.

2. Dulskas A, Smolskas E, Kildusiene I, Samalavicius NE. Treatment possibilities for low anterior resection syndrome: a review of the literature. Int J Colorectal Dis. 2018;33:251-60.

3. Croese AD, Lonie JM, Trollope AF, Vangaveti VN, Ho YH. A Meta-Analysis of the Prevalence of Low Anterior Resection Syndrome and Systematic Review of Risk Factors. Int J Surg. 2018;56:234-41.

4. Chen TY, Wiltink LM, Nout RA, Meershoek-Klein Kranenbarg E, Laurberg $\mathrm{S}$, et al. Bowel function 14 years after preoperative short-course radiotherapy and total mesorectal excision for rectal cancer: report of a multicenter randomized trial. Clin Colorectal Cancer 2015;14:10614.

5. Mortensen AR, ThyøA, Emmertsen KJ, Laurberg S. Chronic pain after rectal cancer surgery-development and validation of a scoring system. Colorectal Dis. 2019;21:90-9.

6. Adam JP, Denost Q, Capdepont M, van Geluwe B, Rullier E. Prospective and Longitudinal Study of Urogenital Dysfunction After Proctectomy for Rectal Cancer. Dis Colon Rectum 2016;59:82230.

7. Ho VP, Lee Y, Stein SL, Temple LK. Sexual function after treatment for rectal cancer: a review. Dis Colon Rectum 2011;54:113-25.

8. Berger N, Ludwig, K, Ridolfi T. Low Anterior Resection Syndrome: Current Management and Future Directions. Clin Colon Rectal Surg. 2016;29:239-45.

9. Emmertsen KJ, Bregendahl S, Fassov J, Krogh K, Laurberg S. A hyperactive postprandial response in the neorectum
- the clue to low anterior resection syndrome after total mesorectal excision surgery? Colorectal Dis. 2013;15:599-606.

10. Zhu X, Lou Z, Gong, H, Meng, R, Hao $\mathrm{L}$, Zhang W. Influence of neoadjuvant chemoradiotherapy on the anal sphincter: ultrastructural damage may be critical. Int J Colorectal Dis. 2016;31:1427-30.

11. Keane C, Wells C, O'Grady G, Bissett IP. Defining low anterior resection syndrome: a systematic review of the literature. Colorectal Dis. 2017;19:713-22.

12. Emmertsen KJ, Laurberg S. Low anterior resection syndrome score: development and validation of a symptom-based scoring system for bowel dysfunction after low anterior resection for rectal cancer. Ann Surg. 2012;255:922-28.

13. López S, Carrillo K, Sanguineti A, Azolas R, Díaz MB, Bocic G, et al. Adaptación transcultural del cuestionario acerca de la función intestinal (LARS Score) para su aplicación en pacientes operados de cáncer de recto medio y bajo. Rev Chil Cir. 2017;69:44-8.

14. Battersby NJ, Bouliotis G, Emmertsen KJ, Juul T, Glynne-Jones R, Branagan J, et al. Development and external validation of a nomogram and online tool to predict bowel dysfunction following restorative rectal cancer resection: the POLARS score. Gut 2018;67:688-96.

15. Itagaki R, Koda K, Yamazaki M, Shuto K, Kosugi C, Hirano A, et al. Serotonin (5-HT3) receptor antagonists for the reduction of symptoms of low anterior resection syndrome. Clin Exp Gastroenterol. 2014;7:47-52.

16. Rosen H, Robert-Yap J, Tentschert G, Lechner M, Roche B. Transanal irrigation improves quality of life in patients with low anterior resection syndrome. Colorectal Dis. 2011;13:e335-8.

17. Martellucci J, Sturiale A, Bergamini C, Boni L, Cianchi F, Coratti A, et al. Rol of transanal irrigation in the treatment of anterior resection syndrome. Tech Coloproctol. 2018;22:519-27.
18. Martellucci J. Low Anterior Resection Syndrome: a treatment algorithm. Dis Colon Rectum 2016;59:79-82.

19. Ramage L, Qiu S, Kontovounisios C, Tekkis P, Rasheed S, Tan E. A systematic review of sacral nerve stimulation for low anterior resection syndrome. Colorectal Dis. 2015;17:762-71.

20. Eftaiha SM, Balachandran B, Marecik SJ, Mellgren A, Nordenstam J, Melich $\mathrm{MD}$, et al. Sacral nerve stimulation can be an effective treatment for low anterior resection syndrome. Colorectal Disease 2017;19:927-33.

21. Vigorita V, Rausei S, Troncoso P, Trostchansky I, Ruano A, Mondaca E, et al. A pilot study assessing the efficacy of posterior tibial nerve stimulation in the treatment of low anterior resection syndrome. Tech Coloproctol. 2017;21:287-93.

22. Altomare DF, Picciariello A, Ferraca C, Digennaro R, Ribas Y, De Fazio M. Shortterm outcome of percutaneous tibial nerve stimulation for low anterior resection syndrome: results of a pilot study. Colorectal Dis. 2017;19:851-6.

23. Powell-Chandler A, Rees B, Broad C, Torkington J, O'Neill C, Cornish JA. Physiotherapy and Anterior Resection Syndrome (PARiS) trial: feasibility study protocol. BMJ open. 2018;8:e021855.

24. Maris A, Devreese AM, D'Hoore A, Penninckx F, Staes F. Treatment options to improve anorectal function following rectal resection: a systematic review. Colorectal Dis. 2013;15:e67-78.

25. Lin KY, Granger CL, Denehy L, Frawley HC. Pelvic floor muscle training for bowel dysfunction following colorectal cancer surgery: A systematic review. Neurourol Urodyn. 2015;34:703-12.

26. Fuentes B, Venegas M. Rol del Kinesiólogo en una unidad de piso pelviano. Revista Médica Clínica Las Condes 2013;24:305-31.

27. Vigorita V, Rausei S, Troncoso P, Trostchansky I, Ruano A, Mondaca E, 
et al. A pilot study assessing the efficacy of posterior tibial nerve stimulation in the treatment of low anterior resection syndrome. Tech Coloproctol. 2017;21:287-93.

28. Visser WS, Te Riele WW, Boerma D, van Ramshorst B, van Westreenen. Pelvic floor rehabilitation to improve functional outcome after a low anterior resection: a systematic review. Ann Coloproctol. 2014;30:109-14.

29. Kye BH, Kim HJ, Kim G, Yoo RN, Cho HM. The effect of biofeedback therapy on anorectal function after the reversal of temporary stoma when administered during the temporary stoma period in rectal cancer patients with sphinctersaving surgery. Medicine 2016;95:e3611.

30. Pucciani F, Ringressi MN, Redditi S, Masi A, Giani I. Rehabilitation of fecal incontinence after sphincter-saving surgery for rectal cancer: encouraging results. Dis Colon Rectum 2008;51:15528.

31. Ridolfi TJ, Berger N, Ludwin KA. Low anterior resection syndrome: current management and future directions. Clin Colon Rectal Surg. 2016;29:239-45.

32. Liang Z, Ding W, Chen W, Wang Z, Du P, Ciu L. Therapeutic evaluation of biofeedback therapy in the treatment of anterior resection syndrome after sphincter-saving surgery for rectal cancer. Clin Colorectal Cancer 2016;15:e 101-7.

33. Silver JK, Baima J. Cancer Prehabilitation: an opportunity to decrease treatment-related morbidity, increase cancer treatment options, and improve physical and psychological health outcomes. Am J Phys Med Rehab. 2013;92:715-27.

34. Carli F, Charlebois P, Stein B, Feldman L, Zavorsky G, Kim DJ, et al. Randomized clinical trial of prehabilitation in colorectal surgery. Br J Surg. 2010; 97:1187-97.

35. Mayo NE, Feldman L, Scott S, Zavorsky G, Kim DJ, Charlebois P, et al. Impact of preoperative change in physical function on postoperative recovery: Argument supporting prehabilitation for colorectal surgery. Surgery 2011;150:505-14

36. Li C, Carli F, Lee L, Charlebois P, Stein B, Liberman AS, et al. Impact of a trimodal prehabilitation program on functional recovery after colorectal cancer surgery: a pilot study. Surg Endosc. 2013;27:107282.

37. Moug SJ, Mutrie N, Barry SJE, Mackay G, Steele RJC, Boachie C, et al. Prehabilitation is feasible in patients with rectal cancer undergoing neoadjuvant chemoradiotherapy and may minimize physical deterioration: results from The REx trial. Colorectal Dis. 2019;21:548-62.

38. West MA, Loughney L, Lythgoe D, Barben CP, Sripadam R, Kemp GJ, et al. Effect of prehabilitation on objectively measured physical fitness after neoadjuvant treatment in preoperative rectal cancer patients: a blinded interventional pilot. Br J Anaesth. 2015;114:244-51.

39. Singh F, Newton RU, Baker MK, Spry NA, Taaffe DR, Galvao DA. Feasibility and efficacy of presurgical exercise in survivors of rectal cancer scheduled to receive curative resection. Clin Colorectal Cancer 2017; 16:358-65.

40. Kim JK, Jeon BG, Song YS, Seo MS, Kwon YH, Park JW, et al. Biofeedback therapy before ileostomy closure in patients undergoing sphincter-saving surgery for rectal. Ann Coloproctol. 2015;31:138-43.

41. Van der Heijden JAG, Thomas G, Caers F, van Dijk WA, Slooter GD, MaaskantBraat AJG. What you should know about the low anterior resection syndrome e Clinical recommendations from a patient perspective. Eur J Surg Oncol. 2018;44:1331-37. 